\title{
Experimental and Numerical Investigation of a Multi-Injector GOX-GCH4 Combustion Chamber
}

\author{
By Simona Silvestri, ${ }^{1)}$ Christoph Kirchberger,${ }^{2)}$ Gregor Schlieben, ${ }^{1)}$ Maria Palma Celano, ${ }^{1)}$ \\ and Oskar HAIDN ${ }^{1)}$ \\ ${ }^{1)}$ Technical University of Munich, Munich, Germany \\ ${ }^{2)}$ German Aerospace Center, Lampoldshausen, Germany
}

(Received June 20th, 2017)

\begin{abstract}
In the current study results from an experimental investigation on an oxygen/methane multi-injector combustion chamber are presented. They provide detailed information about the thermal loads at the hot inner walls of the combustion chamber at representative rocket engine conditions and pressure ranges up to $40 \mathrm{bar}$. The present study aims to contribute to the understanding of the thermal transfer processes and of the interaction between the injectors and the injector-wall. Furthermore, the test results are used as a test case for the validation of the in-house engineering tool Thermtest. Due to the complex flow phenomena linked to the use of cryogenic propellants, like extreme variation of flow properties and steep temperature gradients, in combination with intensive chemical reactions, the problem has been partially simplified by injecting the propellants in gaseous form.
\end{abstract}

Key Words: Green Propellants, Methane, Injector, Efficiency

\section{Nomenclature}

$\begin{array}{cll}\text { A } & : \text { parameter } \\ \mathrm{B} & : \text { parameter } \\ \mathrm{C} & : \text { parameter } \\ \mathrm{c}^{*} & : \text { characteristic velocity } \\ \mathrm{GCH} 4 & : \text { gaseous methane } \\ \mathrm{GGOX} & : \text { gaseous oxygen } \\ \mathrm{J} & : \text { momentum flux ratio } \\ \mathrm{L}^{*} & : \text { characteristic chamber length } \\ \mathrm{LOX} & : \text { liquid oxygen } \\ \dot{m} & : \text { mass flow rate } \\ \mathrm{P} & : \text { pressure } \\ \dot{Q} & : \text { heat rate } \\ \mathrm{r} & : \text { radial distance } \\ \mathrm{T} & : \text { wall temperature } \\ \mathrm{u} & : \text { velocity } \\ \mathrm{VR} & : \text { velocity ratio } \\ \mathrm{Z} & : \text { axial distance } \\ \alpha & : \text { heat convection coefficient } \\ \eta & : \text { combustion efficiency } \\ \theta & : \text { azimuthal distance } \\ \text { cipts } & & \\ \mathrm{hg} & : \text { hot gas } \\ \text { th } & : \text { throat } \\ \text { theo } & : \text { theoretic } \\ \text { tot } & : \text { total }\end{array}$

\section{Introduction}

Recent investigations in Europe on future space transportation have shown a rising interest in $\mathrm{LOX} /$ methane propellants combination for liquid booster stages. ${ }^{1)}$ Methane in fact is favorite for its properties and features among other hydrocarbons. ${ }^{2)}$ In general, this propellant is an interesting candidate to answer the requirements of cost reduction, required performance and environmental aspects. For this reason, several research teams in the US Space program have started comprehensive studies on this propellant and its combination with LOX. ${ }^{3)}$ In Japan, methane has been adopted as the preferred hydrocarbon candidate for the second stage of the J-II vehicle. ${ }^{4)}$ In China LOX/methane combination is under study to develop rocket engine reusability technology.5) European with the prototype engine Prometheus and a cooperation program with Russian industries ${ }^{6)}$ to conceive a LOX/GCH4 engine for booster applications. Purdue University $^{7}$ focuses the attention on LOX/CH4 for expander cycle engines.

Although many studies have already been performed, the engine technology to be employed for new generation launchers lacks an adequate technology maturity, when compared to the well-established cryogenic propellant combinations like liquid oxygen/liquid hydrogen. Because the combustor performance of liquid rocket engines is strongly influenced by both the geometry of the injector and the injector interactions, their design is crucial in the rocket engine development.

In the context of the national research program Transregio SFB/TR-40 on "Technological Foundation for the design of thermally and mechanically high loaded components of Future Space Transportation System", a multi-injector combustion chamber has been designed for gaseous oxygen (GOX) and gaseous methane (GCH4) focusing on high pressure (up to 100 bar) and film cooling behavior. One of the key aspects of the 
project is to improve the knowledge on heat transfer processes and cooling methods in the combustion chamber, which is mandatory for the engine design. The attention is focused, in particular, on injector-injector and injector-wall interaction. In order to have a first characterization of the injector behavior the multi-element combustion chamber is tested at low combustion chamber pressures and for a wide range of mixture ratios. Preliminary test results have been presented in Silvestri et al. ${ }^{8)}$

Detailed results about the circumferential heat load distribution and performance characteristics are the focus of the current paper. Additionally, the test data are used as a test case for the validation of the in-house engineering tool Thermtest. ${ }^{9)}$ Results of the wall heat flux distribution as well as the comparison with the test data are presented.

\section{Test Specimen}

All the experiments have been performed at the Institute of Flight Propulsion's test facility at the Technical University of Munich (TUM). The test bench allows experiments with gaseous methane and gaseous oxygen for interface pressures up to 100 bar. In this section, a brief description of the multi element rocket combustion chamber and the measurement equipment are presented.

\subsection{Hardware description}

The multi-element rocket combustion chamber features an inner diameter of $30 \mathrm{~mm}$, a contraction ratio of 2.5 and is designed for pressure levels up to 100 bar.

In order to easily scale the chamber with the injector dimensions, the distance between the injectors and the injector-wall distance is kept constant and equal to half of the injector diameter, which leads to a pattern of seven injector elements. The combustion chamber, depicted in Fig. 1, comprises mainly four cylindrical water cooled chamber segments, one long and three short segments, for an overall length of $341 \mathrm{~mm}$ and a characteristic chamber length $\left(\mathrm{L}^{*}\right)$ of $906 \mathrm{~mm}$.

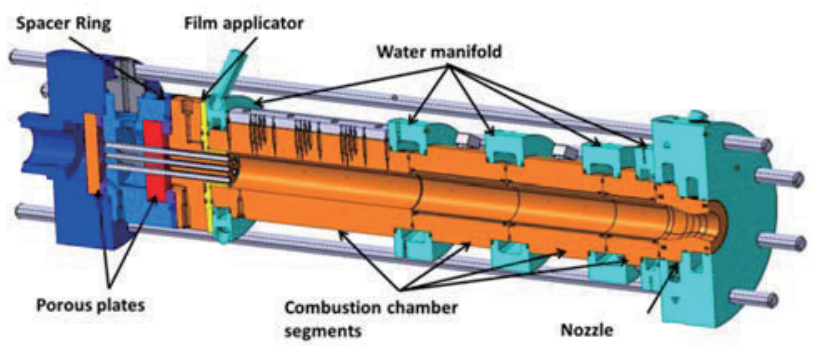

Fig. 1. Combustion chamber schematic.

The injector head of the combustor is designed to allow different injector designs. For the current study, shear coaxial injector elements are mounted. For simplicity as initial configuration the GOX post is mounted flush with respect to the injection plane. In order to center the GOX post in the faceplate, the injector is equipped with four equally-spaced fins. The fins to center the oxygen tubes in the methane annulus are oriented in a radial direction, pointing towards the central injector. In Fig. 2 and Table 1 the main injector characteristic dimensions are reported. To ensure homogeneous injection conditions, in terms of temperature and pressure, two porous plates are placed in the oxidizer and fuel manifold respectively.

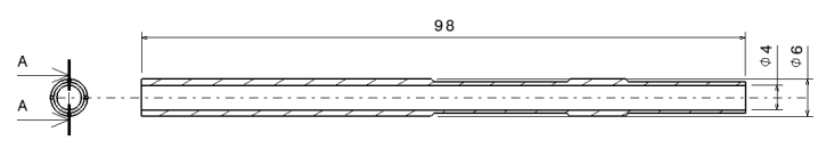

Fig. 2. Injector element.

Table 1. Injector dimensions.

\begin{tabular}{lcr}
\hline GOX post inner diameter $D_{i}$ & {$[\mathrm{~mm}]$} & 4 \\
GOX post wall thickness $w$ & {$[\mathrm{~mm}]$} & 0.5 \\
GOX post recess length & {$[\mathrm{mm}]$} & 0 \\
GCH4 annulus outer diameter $D_{o}$ & {$[\mathrm{~mm}]$} & 6 \\
GOX post length & {$[\mathrm{mm}]$} & 98 \\
Injector area ratio $A_{C H 4} / A_{G O X}$ & {$[-]$} & 0.7 \\
\hline
\end{tabular}

\subsection{Experimental setup and operating conditions}

The experimental setup is equipped with standard instrumentations required to characterize the operation of the chamber. A pattern of pressure transducers provides measurement of the wall pressure distribution along the chamber axis and gives information about the acceleration of the hot gas and hence on the position of the main heat release zone. The pressure transducers of WIKA A10 type are calibrated before each test campaign, therefore only a no repeatability error of maximum 0.06 bar is considered for the calculations.

To describe the injection conditions, temperature and pressure of the injected propellants are recorded in the manifolds after the porous plates. To determine the temperature field, the chamber segments are equipped with 90 type $\mathrm{T}$ thermocouples spring-mounted in the chamber wall. The spring loading of the thermocouples provides a constant force to ensure a continuous contact between the thermocouples tip and the base of the hole. ${ }^{10)}$ This setup aims to minimize the chance of potential loss of contact caused by expansion and contraction of the material due to changes in temperature or vibrations during the hot run. In the first segment the thermocouples are mounted on four azimuthal positions to monitor the behavior of two adjacent external injectors and the one positioned at the center of the chamber, as shown in Fig. 3. Moreover, a regular pattern along the chamber axis provides information about the progress of combustion.

The mass flow rates in the combustion chamber are determined by regulation valves and measured during the test by Coriolis flow meters. An accuracy of $0.1 \%$ of the mass flow rate is considered. Since the mass flow rates are influenced by the upstream pressure and temperature, prior to each test flow checks are required to set an accurate opening of the valves. For the design of the operating points, the characteristic velocity $\mathrm{c}^{*}$ is calculated with $\mathrm{CEA} 2^{13)}$ and a combustion efficiency of $\eta \mathrm{c}^{*}=1$ is assumed. 


\begin{tabular}{cc}
\hline Position & Angle $\left[^{\circ}\right]$ \\
12 & 0 \\
01 & 30 \\
02 & 60 \\
03 & 90 \\
04 & 120 \\
05 & 150 \\
06 & 180 \\
07 & 210 \\
08 & 240 \\
09 & 270 \\
10 & 300 \\
11 & 330 \\
\hline
\end{tabular}

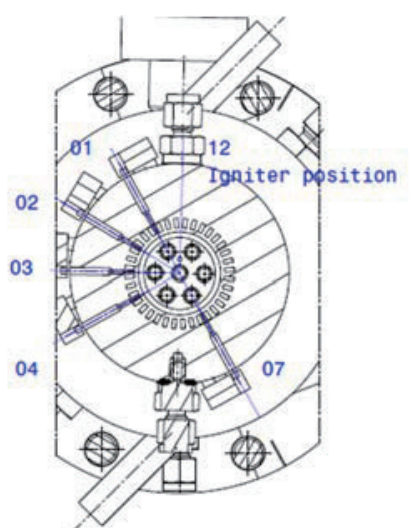

Fig. 3. Azimuthal thermocouple schematic in the first chamber segment.

A typical test sequence includes one pressure level with three different mixture ratios. The burn time of the combustion chamber is chosen to reach stable operation, required for the thermal load measurements. To minimize the water consumption of the cooling system, a stable run time of $40 \mathrm{~s}$ is chosen. The experimental data presented are the average values over the time intervals $\mathrm{t} 1, \mathrm{t} 2$ and $\mathrm{t} 3$ given in Fig. 4. To establish stable set point conditions (Pc, OF) and reach thermal stabilization, time intervals of $1 \mathrm{~s}$ have been chosen. The test matrix comprises tests at pressure levels from nominally 20 up to 40 bar and mixture ratios of 2.6, 3.0, 3.4.

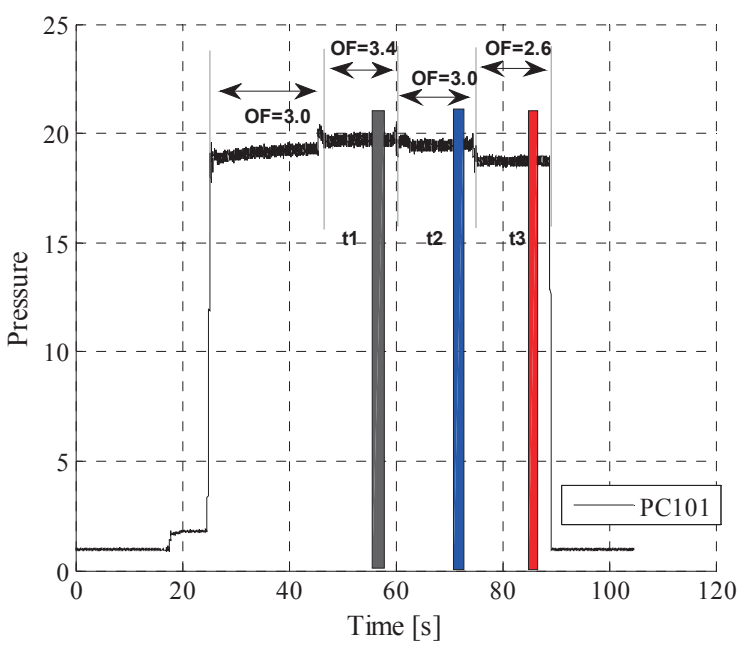

Fig. 4. Pressure signal over time.

\section{Experimental Results}

In a shear coaxial injector, the mixing efficiency is mainly driven by the shear forces between the propellants, therefore two non-dimensional numbers are employed to characterize the injection conditions. The velocity ratio (VR) between the fuel and the oxidizer stream velocity is defined in Eq. (1), and the momentum flux ratio $(\mathrm{J})$ is calculated as the ratio of the fuel momentum to the oxidizer momentum in Eq. (2).

$$
V R=\frac{u_{G C H 4}}{u_{G O X}}
$$

and

$$
J=\frac{\left(\rho u^{2}\right)_{G C H 4}}{\left(\rho u^{2}\right)_{G O X}}
$$

For the test performed, the values of the VR and $\mathrm{J}$ are given in Fig. 5 and Fig. 6. Due to the gaseous form of the propellants, both ratios show a linear dependency on the decreasing mixture ratio and no clear dependency on the pressure level in the tested range. All calculated values are based on propellant temperature and pressure at injection conditions. While the fluid properties have been derived by REFPROP, ${ }^{12)}$ the injection velocity is determined by the propellant mass flow during the time interval considered.

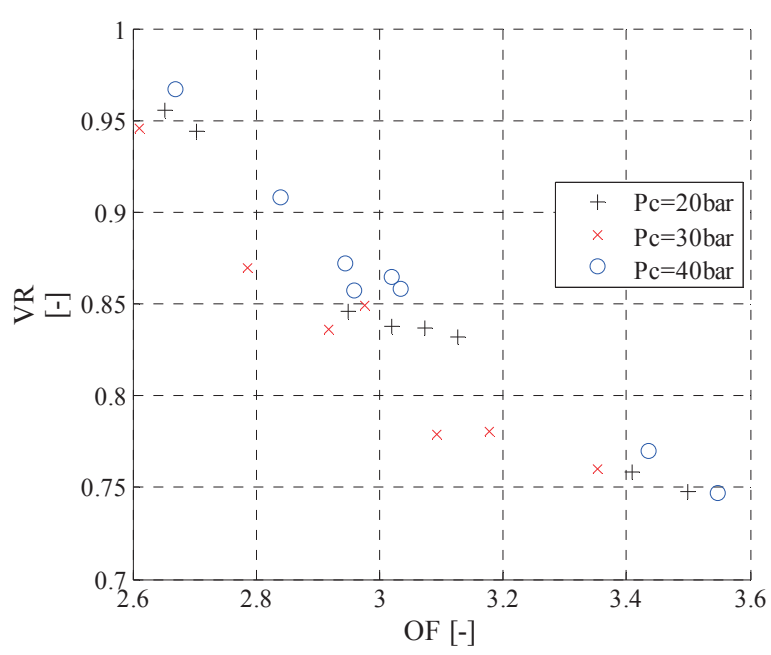

Fig. 5. Velocity ratio vs OF.

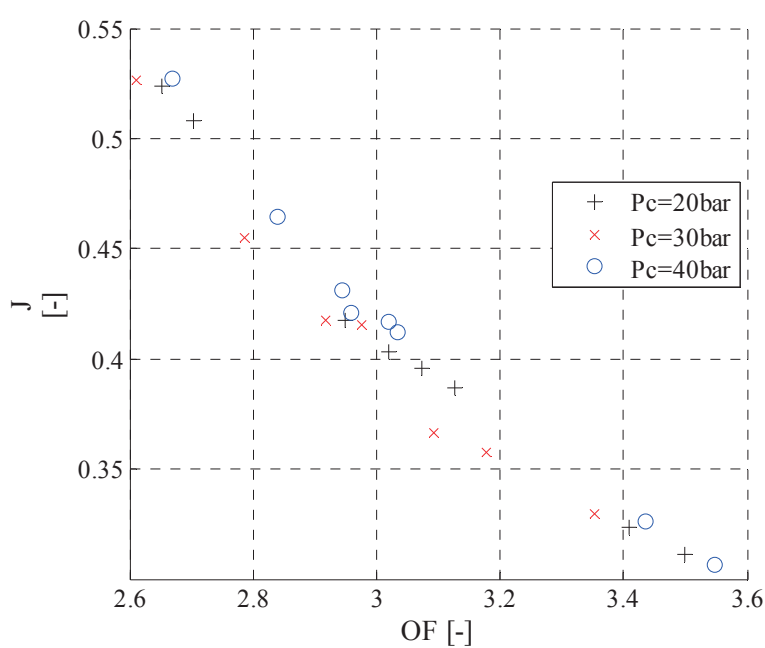

Fig. 6. Momentum flux ratio vs OF.

To fully characterize the behavior of the shear coaxial injector, $\mathrm{Cd}$ values have been calculated for all the load points. Because it is not possible to measure the fluid temperatures directly at the entry to the combustion chamber, injection conditions are calculated based on the first pressure sensor in 
the combustion chamber and the corresponding manifold temperatures. With this approach, heat transfer between the propellants, both at ambient temperature, within the injection element is considered negligible. Figure 7 shows the $\mathrm{Cd}$ values of the methane annulus, in red, and of the oxygen tube, in blue, over the variation of the injection Reynolds number. The Reynolds number of both propellant sides is in a fully development turbulent regime. No strong influence can be observed from changes in neither mixture ratios nor combustion chamber pressure. A relative error of \pm 0.06 due to the measurement uncertainty has to be taken into account.

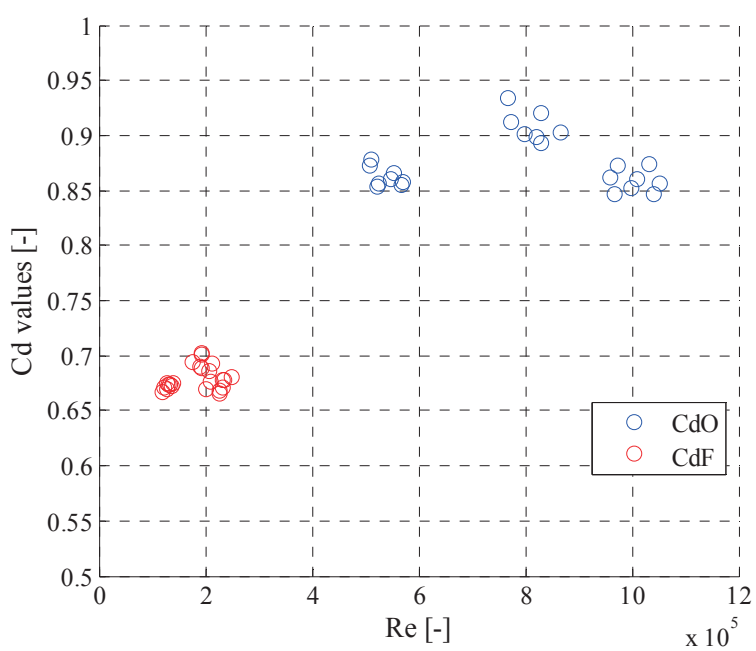

Fig. 7. Discharge coefficients.

\subsection{Azimuthal temperature distribution}

Detailed knowledge of the thermal loads is essential for an optimal design of the cooling system and engine cycle. Although the thermal loads are usually lower in the near injector region compared to the throat area, this area is quite important since characterized by not fully mixed propellant jets with consequent inhomogeneity of velocity and species concentrations. Moreover, to understand the injector-injector interaction and the foot print of the injectors on the chamber wall, a detailed look of the temperature distribution in azimuthal direction is necessary. The difference between the temperature value in the first chamber segment at each radial, axial and azimuthal position and the mean temperature value in circumferential direction for the thermocouples placed at the same radial distance from the hot wall, is define as in Eq. (3):

$$
T_{\theta}=\frac{T\left(r, \theta_{i}, z\right)-\frac{1}{n} \sum_{i}^{n} T\left(r, \theta_{i}, z\right)}{\frac{1}{n} \sum_{i}^{n} T\left(r, \theta_{i}, z\right)} * 100
$$

where $\mathrm{n}$ is the number of temperature measurements available in circumferential direction.

Figure 8 shows the temperature difference $T_{\theta}$ for the pressures and mixture ratios tested. For a better visualization of the temperature field, the azimuthal positions at $60^{\circ}$ and $90^{\circ}$ are mirrored in the azimuthal position $0^{\circ}$ and $150^{\circ}$. It can be seen that the azimuthal temperature distribution is not completely symmetric. The temperature value at $30^{\circ}$ is showing the maximum temperature, which decreases proceeding in circumferential direction. Although each injector has been precisely manufactured and features the same geometric characteristic, this kind of behavior is visible for all the tests performed. A reason for the circumferential asymmetry is still under investigation and a possible source has been initially attributed to the energy released by the ignition system, positioned at $0^{\circ}$ in the faceplate, see Fig. 3. However after a detailed analysis of all azimuthal temperature distribution data we can exclude such an impact.

The angular positions $30^{\circ}$ and $90^{\circ}$ are aligned to the positions of the central axes of the nearby injector elements in the outer row. At these two angular positions, the temperature appears to be higher than in the angular position between the injectors $\left(60^{\circ}\right.$ and $\left.120^{\circ}\right)$. This kind of behavior has already been encountered for hydrogen/oxygen flame in multi-element chambers. ${ }^{11)}$ Within an axial distance of $80-100 \mathrm{~mm}$, this behavior changes and the wall temperature at angular positions between the injectors, at $60^{\circ}$ and $120^{\circ}$, becomes higher. In the near injector region, the flames are not fully developed and the initial hydrodynamic mixing of the propellants plays a big role. Here the interaction between the injectors is not strong, each injector can be mainly considered as independent. Hence, the maximum temperature would be achieved at the angular position where the injectors are located. Proceeding downstream towards the nozzle, the flames expand, the interaction becomes more intense and the injector placed in the center would contribute to the heat release footprint towards the chamber walls. Even though no substantial differences can be seen with variation of mixture ratio, the form and the intensity of the distribution vary with the increase in chamber pressure. The higher the chamber pressure, the higher is the temperature difference between the angular position corresponding to the injector and the angular position between the injectors. This characteristic footprint of the injection system remains visible within the first $60-80 \mathrm{~mm}$. The majority of the heat release takes place in this region, as shown in previous publications. ${ }^{8)}$ While close to the faceplate the flame shape is strongly dependent from the injection conditions where no large scale mixing in the near wall region is visible, more downstream, when the heat release has reached a certain critical level, a dependence on pressure level is noticeable. Indeed, a variation in the flame shape is visible after an axial position of around $100 \mathrm{~mm}$ with increasing chamber pressure. This variation is consistent for each test repetition at the same combustion chamber pressure and appears very similar for each mixture ratio tested.

For all the load points tested, the relative error $(\delta \mathrm{T} \theta / \mathrm{T} \theta)$ is smaller than 0.15 . The error calculation is based only on the measurement tolerance of the type $\mathrm{T}$ thermocouples of $0.5 \mathrm{~K}$. Further deviations due to the accuracy in the thermocouples installations are neglected. Since the same hardware setup is used, these deviations do not influence the comparison between the different tests performed. 


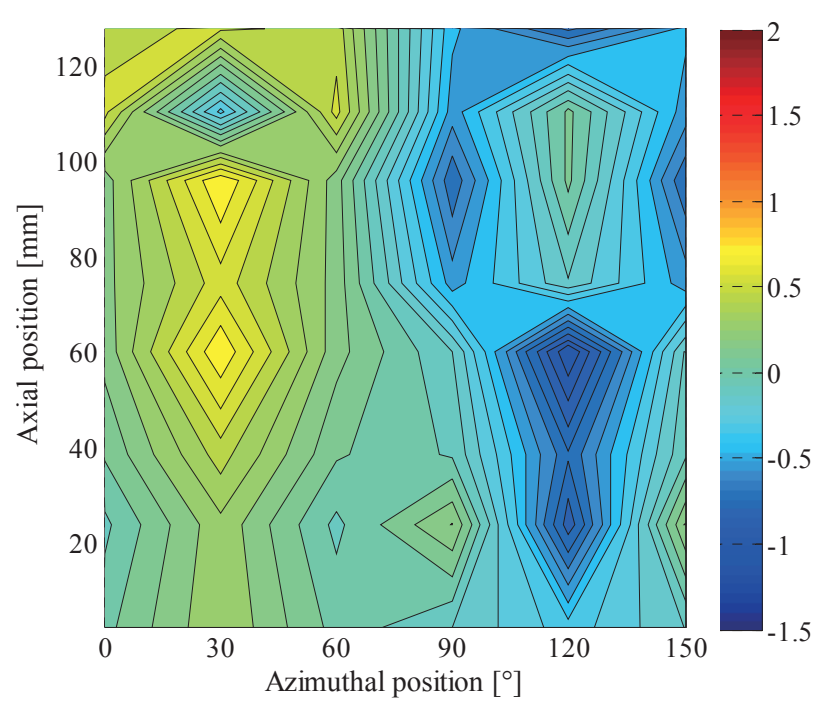

(a) $\mathrm{Pc}=20$ bar, $\mathrm{OF}=2.6$

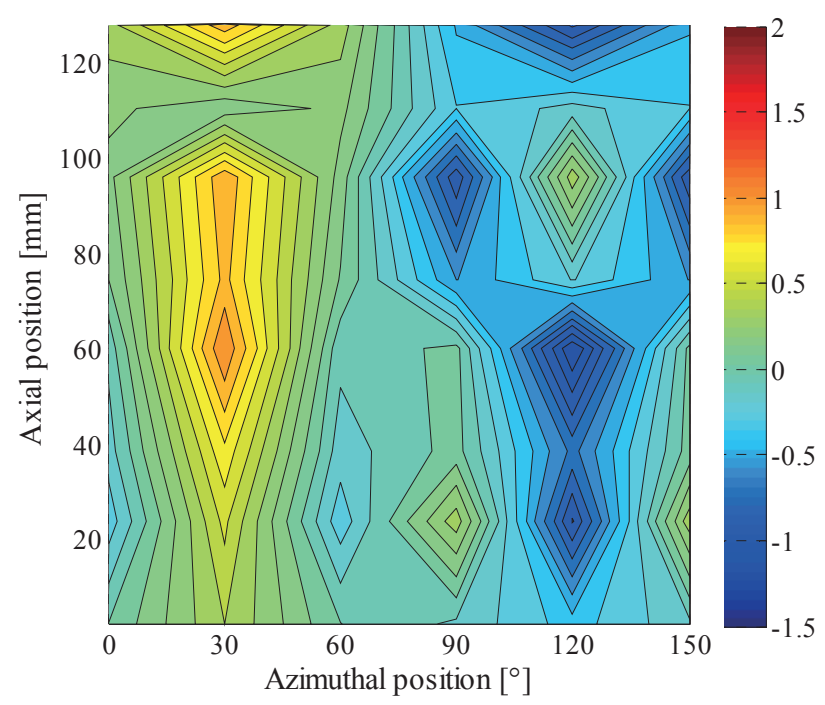

(c) $\mathrm{Pc}=30$ bar, $\mathrm{OF}=2.6$

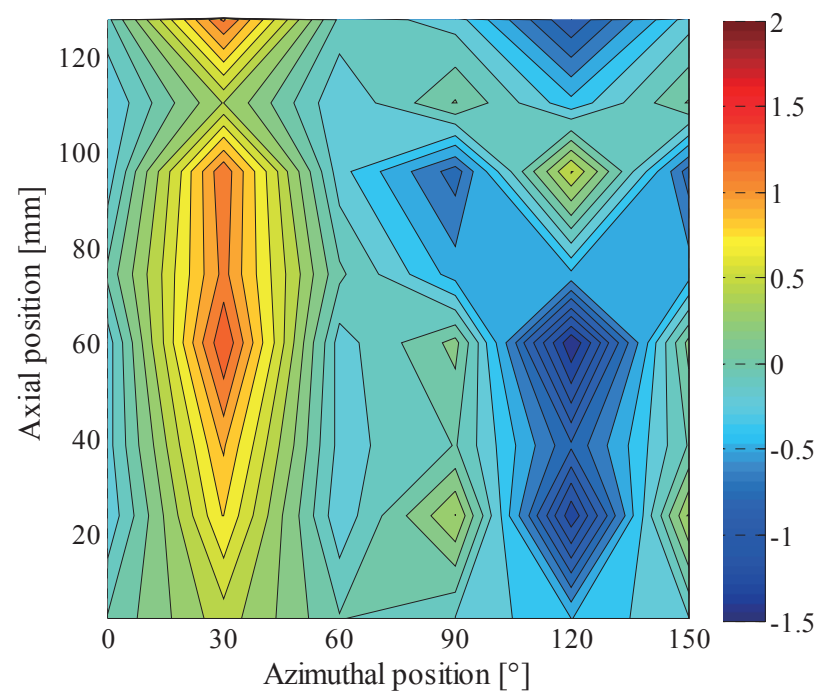

(e) $\mathrm{Pc}=40$ bar, $\mathrm{OF}=2.6$

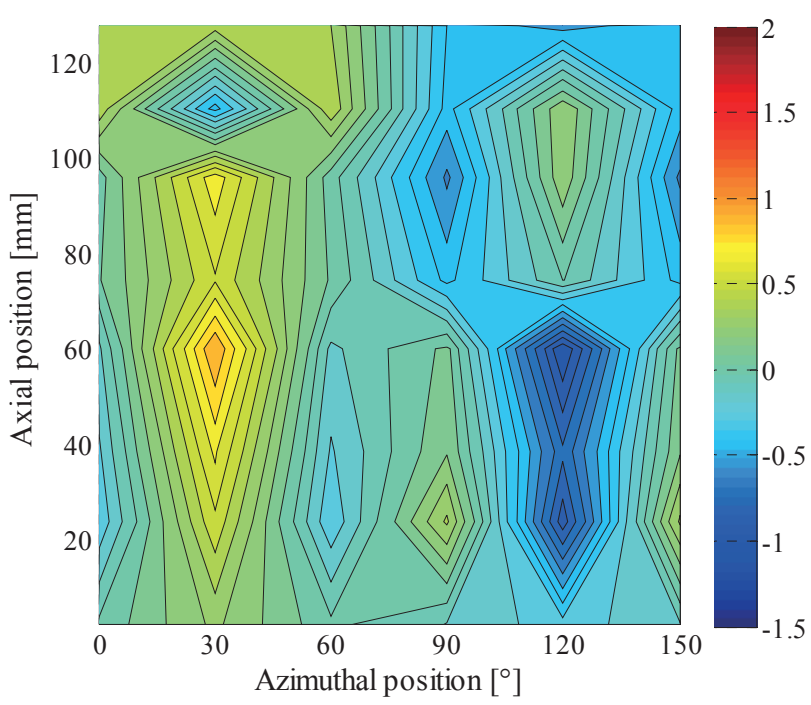

(b) $\mathrm{Pc}=20$ bar, $\mathrm{OF}=3.4$

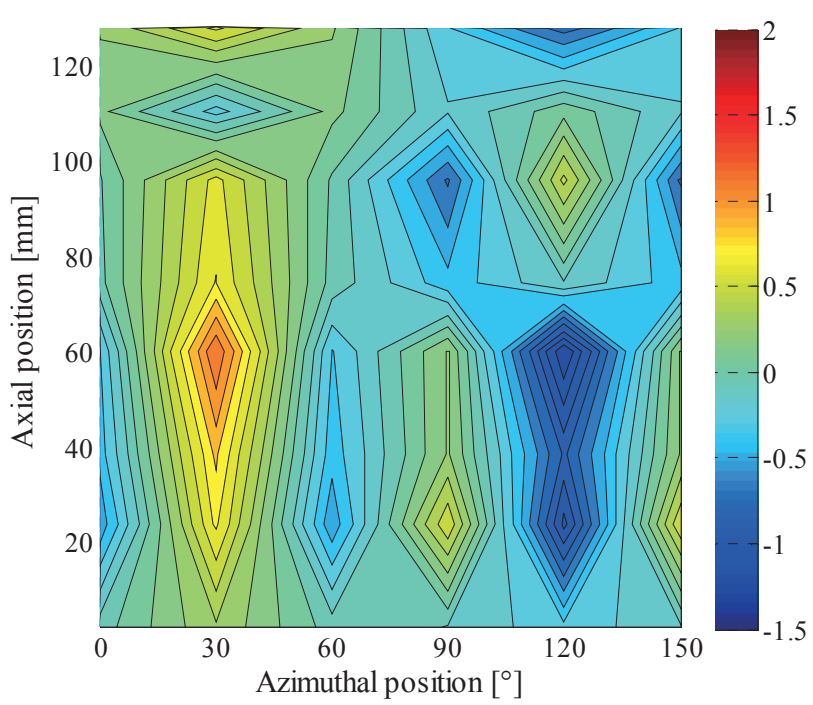

(d) $\mathrm{Pc}=30$ bar, $\mathrm{OF}=3.4$

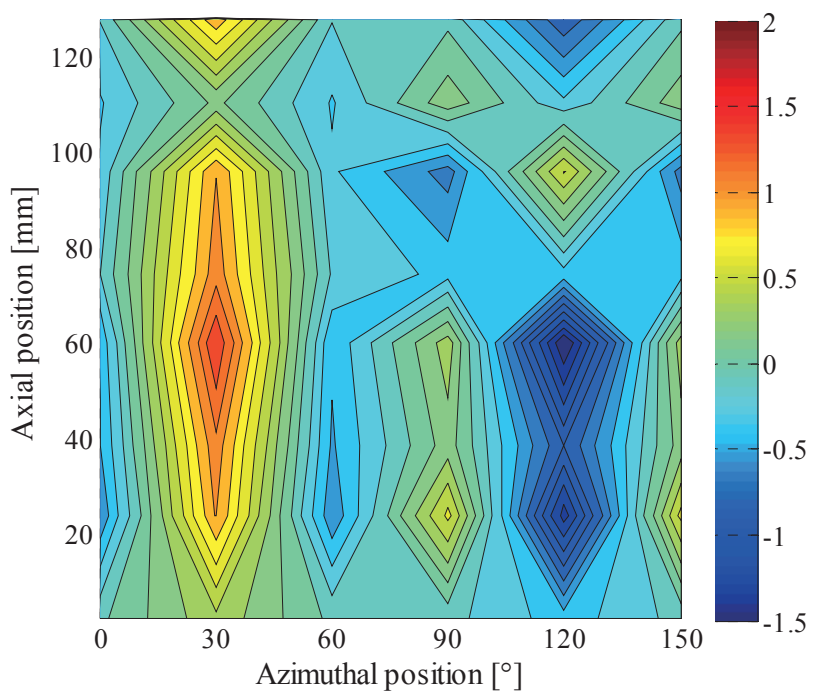

(f) $\mathrm{Pc}=40$ bar, $\mathrm{OF}=3.4$

Fig. 8. Distribution of the azimuthal temperature difference for different pressures and mixture ratios (a)-(f). 


\subsection{Axial distribution of thermal loads}

For the determination of the thermal loads a calorimetric method is applied. The heat rate to each chamber segment is determined by the balance of the coolant total enthalpy from inlet to outlet. Therefore, precise temperature and pressure measurements are located in the water manifolds between the test segments. The coolant mass flow is determined by the measure of the differential pressure over metering orifices. The integral heat rate is defined as in Eq. (4).

$$
\dot{Q}=\dot{m}_{\text {water }}\left(h_{\text {out }}\left(T_{\text {out }}, P_{t, \text { out }}\right)-h_{\text {in }}\left(T_{\text {in }}, P_{t, \text { in }}\right)\right)
$$

Fluid properties are derived by Refprop, ${ }^{12)}$ while the enthalpy definition is calculated according to Gordon-McBride. ${ }^{13)}$ Since the determination of the heat flux is quite sensitive to measurements uncertainties, the chamber water manifolds, as well as the nozzle segment water manifold are equipped with multiple thermocouples placed in azimuthal direction. The circumference of the chamber is divided in twelve sectors. For each sector the individual heat pick-up is calculated. The heat flux is then obtained by normalization of the mean value of the heat pick-up to the inner surface area of each corresponding segment. A detailed analysis of the heat flux results for all the load points can be found in Silvestri et al. ${ }^{8)}$

Using the calorimetric technique, global heat transfer properties can be determined with good accuracy. However, local behavior of heat transfer cannot be resolved. For this reason, simulation with the in house predicting tool, Thermtest are ongoing at the institute. Thermtest allows the simulation of steady as well as transient thermal behavior of cooled or uncooled structures over a wide scope of chamber materials and cooling fluids. While the heat conduction inside the chamber material is solved by a 3D finite difference method, the convective heat transfer from the hot gas side to the inner wall as well as for the coolant is modeled using empirical Nusselt correlations. The hot wall heat transfer coefficient is, for the case used, calculated from a modified formulation proposed by Sinyarev. ${ }^{14)}$ General information about Thermtest can be found in Kirchberger et al. ${ }^{15)}$

In the past, Thermtest has been validated and adapted for the use of kerosene/oxygen as propellants in small single-element combustion chambers featuring swirl or double swirl coaxial injector elements ${ }^{16}$ ) and for a single element capacitive combustion chamber using methane/oxygen. ${ }^{17)}$ Efforts have been done to further validate the tool for methane/oxygen propellant combination and to adapt the empirical correlations to the injector behavior. Based on the work of Ponomarenko, ${ }^{18)}$ a correction function for the heat load characteristic of generic coaxial injector elements has been applied, as shown in Eq. (5). Here, the hot gas heat convection coefficient is described by means of three parameters: A, B and C. In order to account for the reduced heat transfer at the beginning of the combustion chamber, where the combustion evolves, the heat flux shape is prescribed as a function of the axial coordinate $\mathrm{z}$.

$$
\alpha_{h g, \mathrm{corr}}=A \alpha_{h g}\left[(1-B)+B \operatorname{tangh}\left(\frac{Z}{C} \pi\right)\right]
$$

An optimization process that minimize the difference in predicted and measured heat flux as well as predicted and measured wall temperature has been carried out in order to establish the optimum set of parameters for the test data. Preliminary results of the heat flux for the 20 bar test $\mathrm{OF}=3.0$, obtained with the above mentioned optimization procedure, are shown in Fig. 9.

Although the simple form of Eq. (5), a good agreement is visible between the test data, in red, and the simulated integral heat flux, in dark blue dashed line. The determination of thermal physical properties of the combusted gases in the boundary layer and the changes of these properties due to variation of hot gas temperature, pressure and mixture ratio is a challenging topic for the research community and can be rarely achieved without the adoption of a finite rate chemistry approach. Nevertheless the heat flux profile for coaxial injector appears to be very distinctive and can be well approximated by the rather simple model presented.

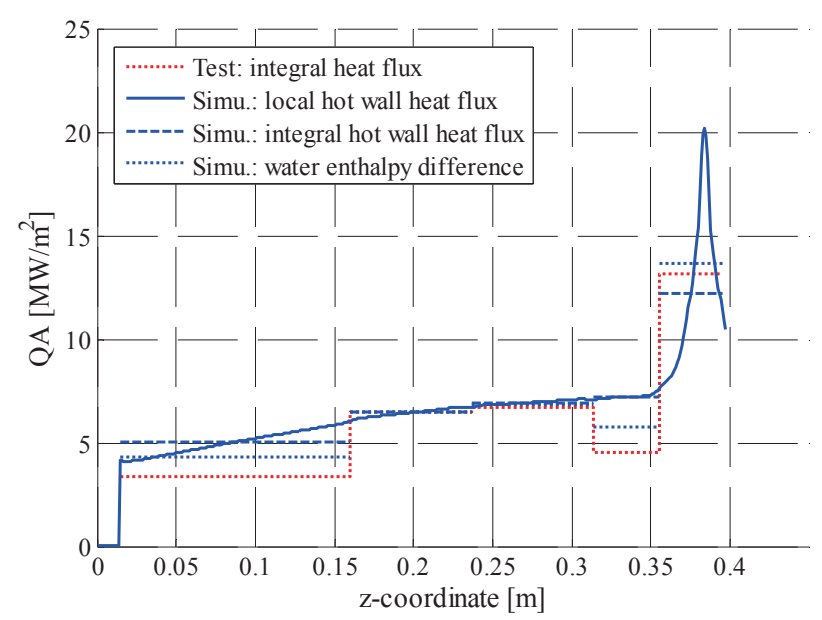

Fig. 9. Axial heat flux distribution, simulations and experiments for the $20 \mathrm{bar} \mathrm{OF}=3.0$ test case.

Even though from the first to the second segment an increase in heat flux of $100 \%$ is recognizable in the test data, only a slight increase in heat flux is visible from the second to the third segment. This indicates that the combustion can be considered largely completed until the end of the second segment. In this region, the heat fluxes from the simulation and experiments show a good match with a maximum deviation of $2.9 \%$. A discrepancy of $26 \%$ and $50 \%$ is instead visible in the first and last chamber segment, respectively. The deviation in the last chamber segment and nozzle is easily explainable when looking at the water enthalpy difference in the simulations (dark blue dotted line). It appears in fact that the difference of the water mass flow rate used for cooling ( $\dot{m}_{\text {water, nozzle }} \sim 2 \mathrm{~kg} / \mathrm{s}$ and $\dot{m}_{\text {water,chamber }} \sim 0.5 \mathrm{~kg} / \mathrm{s}$ for the test under analysis), produces a strong axial thermal exchange between the two water cooled segments. This would explain the decrease in heat flux in the fourth segment observable for all the tests performed. This trend is well captured by the simulations and reduces the discrepancy in the fourth segment to $21 \%$. Therefore, future optimization process will aim to optimize the experimental 
heat flux with the enthalpy difference predicted from the simulations. This would allow decoupling the effect of the thermal exchange between nozzle and chamber and characterizing other effects coming from the hot gas side, as for example boundary layer growth in this area, that could also cause a reduction of the heat flux measured.

The source of the overestimation of the heat flux in the first chamber segment is not fully understood and still under investigation. On one hand the imposed initial value of the heat flux could generate a not realistic too high starting value of the heat flux at the faceplate, on the other hand the shape imposed by the correction does not allow a local faster growth of the profile. Both will result in a higher value of the heat flux predicted with Thermtest.

\subsection{Combustion efficiency}

To describe the performance of an injector system one of the parameters to look at is the combustion efficiency. The combustion efficiency is a measure of the effectiveness of a combustion chamber to convert the internal energy contained in the propellants into heat energy and may be defined as the experimental characteristic velocity, $c_{\text {exp }}^{*}$, divided by the theoretical (complete-combustion) characteristic velocity, $c_{\text {theo }}^{*}$, Eq. (6).

$$
\eta_{c^{*}}=\frac{c_{\text {exp }}^{*}}{c_{\text {theo }}^{*}}
$$

The experimental characteristic velocity is defined as in Eq. (7):

$$
c^{*}=\frac{p_{t o t} A_{t h}}{\dot{m}_{t o t}}
$$

Here, $p_{t o t}$ is the total pressure in the throat, $A_{t h}$ is the throat area and $\dot{m}_{\text {tot }}$ is the total propellant mass flow rate injected in the combustion chamber. A theoretical calculation, performed with CEA2, is used to evaluate the theoretical characteristic velocity. The properties and the enthalpy of the propellants, the mixture ratio and the combustion chamber pressure are the inputs for the calculation. The combustion chamber pressure to be used as input is iteratively computed by minimizing the error between the theoretical static pressure calculated at the "combustion-end" in Gordon and McBride with the experimental wall pressure measured shortly before the nozzle section.

To have a correct estimation of the combustion efficiency, the heat losses towards the combustion chamber wall must be considered in the adiabatic CEA2 computation. To take into account the non-adiabatic wall, a correction of the initial propellants' energy is made.

In the scope of this work two approaches are followed to calculate the experimental characteristic velocity, the LFA-Legacy and the Jannaf method. The two methods differ in the procedure used to calculate the total combustion chamber pressure in the throat section. While the LFA-Legacy method assumes equilibrium conditions to calculate the heat capacity ratio, the Jannaf method assumes frozen condition at the "combustion-end". However, the difference in combustion efficiency computed with the two approaches is usually lower than $0.1 \%$. The combustion efficiency calculated with the Jannaf procedures with respect to the velocity ratio is given in Fig. 10. While the various pressure ranges are plotted with different symbols, the different mixture ratios are represented by different colors.

Although no clear influence of the variations in combustion chamber pressure is noticed, a clear trend is recognizable with variations in mixture ratios. Indeed, approaching lower mixture ratio the combustion efficiency tends to decrease. Several factors may contribute to these results.

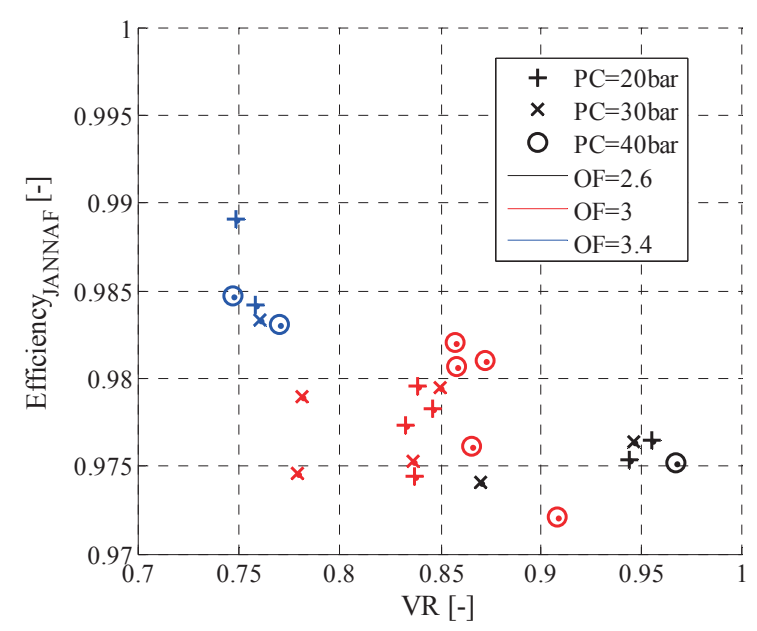

Fig. 10. Combustion efficiency.

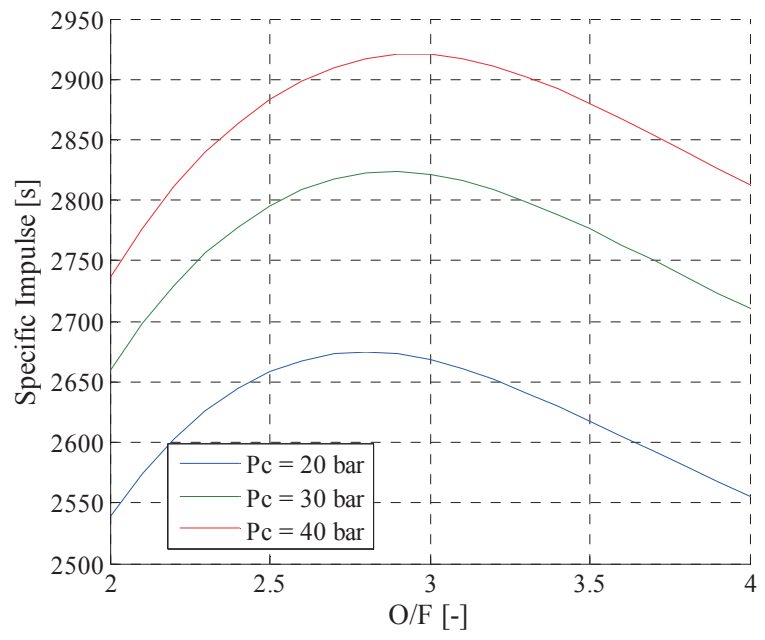

Fig. 11. Specific impulse.

A typical range of mixture ratios used in rocketry for methane/oxygen combustion is given in Fig. 11. To achieve higher specific impulse and diminish contact between the high temperature oxygen and the chamber wall material, only fuel rich cases are usually considered. Therefore an increase in mixture ratio will increase the amount of oxygen in the system and lead to more heat release. This influence at the pressure ranges studied is however minimal. 
The main effect of changes in mixture ratios is the variation, although limited, of the velocity ratio between the propellants. The increase of the initial shear forces produces a favorable mixing in the reacting shear layer and has a strong impact on the overall combustion process. Any increase in the shear forces (velocity ratio $\neq$ from 1 ) will result in an increase of the combustion efficiency. Due to the gaseous nature of the propellants, this fluid dynamic effect is independent from the pressure ranges tested, see Fig. 5. To further prove this effect that seems to dominate the whole combustion process, lower mixture ratio will be tested in the future.

The increase of oxygen mass flow rate, for higher mixture ratios, yields an increase of its injection velocity, consequently for the same time difference an oxygen fluid element will be transported further downstream (decrease of ratio of diffusion time to residence time) and thus the entire flame (reaction zone) will be stretched, this however doesn't seem to affect the combustion efficiency, implying a sufficient characteristic length of the hardware.

\section{Conclusion}

In the context of the national research program Transregio $\mathrm{SFB} / \mathrm{TR} 40$ a multi-injector combustion chamber is designed and tested for GOX and GCH4 propellants research purposes. Detailed wall temperature measurements and derived heat flux data sets have been acquired over a range of pressure (20 to 40 bar) and mixture ratio (2.6 to 3.4) conditions. These data sets are valuable for both injector design and to validate the numerical and simulation tools. The azimuthal wall temperature profile has been analyzed in detail. It has been observed that until a certain axial length $(\mathrm{z}=80-100 \mathrm{~mm})$ the injector footprint is not influenced by the variation of combustion chamber pressure or mixture ratio. After this length, where the heat release is more pronounced, a variation with combustion chamber pressure is found. Furthermore, the combustion efficiency has been analyzed. A decrease in efficiency with the mixture ratio has been observed. A first optimization process has been performed with the in-house simulation tool Thermtest. Here the deviation between experimental and calculated heat flux is minimized by means of optimization of the model parameters. Good agreement has been achieved and the local averaged heat flux profile has been determined.

\section{Acknowledgments}

Financial support has been provided by German Research Foundation (Deutsche Forschungsgemeinschaft-DFG) in the framework of the Sonderforschungsbereich Transregio 40. Furthermore, the authors would like to thank Julian Strauss for the help given in the analysis of the test data.

\section{References}

1) Preclik, D., Hagemann, G., Knab, O., Brummer, L., Mäding, C., and Wiedmann, D.: LOX/Hydrocarbon Propellant Trade Considerations for Future Reusable Liquid Booster Engines, AIAA Paper 2005-3567, 2005.

2) Burkardt, H., Sippel, M., Herbertz, A., and Klevanski, J.: Kerosene vs Methane: A Propellant Tradeoff for Reusable Liquid Booster, $J$. Spacecraft and Rockets, 41(2004), pp. 762-769.

3) Locke, J., Pal, S., and Woodward, R.: Chamber Wall Heat Flux Measurements for a LOX/CH4 Propellant Uni-Element Rocket, AIAA Paper 2007-5547, 2007.

4) Ishikawa, Y., Kodama, S., Ishii, M., Higashino, K., Ikeda, H., Arita, M., Arita, G. P., and Mossolov, S. V.: LOX /LNG Engine Design and Test Results for J-II Rocket, AIAA Paper 2000-3454, 2000.

5 Sun, J.: An Experimental Study of LOX/Methane Subscale Injector, IAC, 65th International Astronautical Congress, Canada, IAC-14.C4.3.10, 2014

6 Bonhomme, C., Theron, M., Louaas, E., Beaurain, A., and Seleznev, E.P.: French/Russian Activities on LOX/LCH4 Area, 57th International Astronautical Congress, Valence, IAC-06-C4.3.7, 2006.

7 Schuff, R., Maier, M., Sindily, O., Ulrich, C., and Fugger, S.: Integrated Modeling and Analysis for LOX/Methane Expander Cycle Engine, AIAA Paper 2006-4534, 2006.

8 Silvestri, S., Celano, M. P., Schlieben, G., and Haidn, O. J.: Characterization of a Multi-Injector GOX-GCH4 Combustion Chamber, AIAA Paper 2016-4992, 2016.

9 Kirchberger, C., Schlieben, G., and Haidn, O. J.: Assessment of Analytical Models for Film Cooling in a Hydrocarbon/ GOX Rocket Combustion Chamber, AIAA Paper 2012-3909, 2012

10 Suslov, D., Woschnak, A., Sender, J., and Oschwald, M.: Test Specimen Design and Measurement Technique for Investigation of Heat Transfer Processes in Cooling Channels of Rocket Engines Under Real Thermal Conditions, AIAA Paper 2003-4613, 2003.

11 Suslov, D. I., Arnold, R., and Haidn, O. J.: Investigation of Two Dimensional Thermal Loads in the Region Near the Injection Head of a High Pressure Subscale Combustion Chamber, AIAA Paper 2009-450, 2009.

12 Lemmon, E. W., Huber, M. L., and McLinden, M. O.: NIST Standard Reference Database 23: Reference Fluid Thermodynamic and Transport Properties-REFPROP, Version 9.0, National Institute of Standards and Technology, Standard Reference Data Program, Gaithersburg, 2010.

13 Gordon, S. and McBride, B.: Computer Program for Calculation of Complex Chemical Equilibrium Compositions and Applications, NASA Reference Publication, 1994.

14 Schmidt, G.: Technik der Flüssigkeits-Raketentriebwerke, DaimlerChrysler Aerospace, Munich, 1999.

15 Kirchberger, C., Wagner, R., Kau, H. P., Soller, S., Martin, P. Bouchez, M., and Bonzon, C.: Prediction and Analysis of Heat Transfer in Small Rocket Chambers, AIAA Paper 2008-1260, 2008 .

16 Kirchberger, C., Schlieben, G., Hupfer, A., Kau, H. P., and Soller, S.: Investigation on Film Cooling in a Kerosene/GOX Combustion Chamber, AIAA Paper 2009-5404, 2009.

17 Celano, M. P., Silvestri, S., Schlieben, G., Kirchberger, C., Haidn, O. J., Dawson, T., Ranjan, R., and Menon, S.: Experimental and Numerical Investigation for a GOX-GCH4 Shear-Coaxial Injector Element, Space Propulsion Conference 2014, Cologne, Germany, SP2014_2969417, 2014.

18 Ponomarenko, A: Thermal Analysis of Thrust Chambers, Software Manual, RPA: Tool for Rocket Propulsion Analysis, 2012. 